\title{
Solução Exata e Estabilização Exponencial para a Equação de Allen-Cahn
}

\author{
D. S. ALMEIDA JÚNIOR ${ }^{1}$, A. J. A. RAMOS ${ }^{2}$, L. M. RIBEIRO ${ }^{3 *}$ e E. D. P. TEIXEIRA ${ }^{4}$
}

Recebido em 30 de junho de 2020 / Aceito em 29 de março de 2021

\begin{abstract}
RESUMO. Neste trabalho estudamos algumas propriedades qualitativas da equação de Allen-Cahn. Esta equação tem sido amplamente estudada em diversas áreas da ciência e principalmente na evolução de microestruturas durante o processo de solidificação de um metal puro ou liga metálica. Os principais resultados obtidos são: a solução exata, a energia de Ginzburg-Landau e a propriedade de decaimento exponencial da energia total do modelo. A solução exata do problema foi obtida pelo método da separação de variáveis, graças a uma escolha adequada do coeficiente de reação. Em relação a estabilização exponencial da energia total das soluções, usamos técnicas multiplicativas para estabelecer a lei de dissipação da energia e, em seguida, usamos algumas desigualdades clássicas da análise matemática para construir a estimativa de decaimento exponencial.
\end{abstract}

Palavras-chave: equação de difusão, equação de Allen-Cahn, solução exata, estabilização exponencial.

\section{INTRODUÇÃO}

A evolução de microestruturas durante o processo de solidificação de um metal puro ou liga metálica pode ser estudada com o auxílio de modelos matemáticos de campo de fase "phasefield", que consideram que a transição entre uma fase e outra, ocorre de forma contínua e gradual, através de uma região reduzida chamada interface difusa [5]. Dentre os modelos que utilizam o método de campo de fase, destacamos o modelo de Allen-Cahn [1]. A equação abaixo, postulada por Allen e Cahn

$$
\frac{\partial \phi}{\partial t}=-M \frac{\delta G}{\delta \phi}
$$

\footnotetext{
*Corresponding author: Lindomar Miranda Ribeiro - E-mail: lindomar@ufpa.br

${ }^{1}$ Programa de Doutorado em Matemática, Universidade Federal do Pará, R. Augusto Corrêa, 01, 66075-110, Belém, PA, Brasil - E-mail: dilberto@ufpa.br https://orcid.org/0000-0002-9925-3694

${ }^{2}$ Faculdade de Matemática, Universidade Federal do Pará, R. Raimundo Santana Cruz, s/n, 68721-000, Salinópolis, PA, Brasil - E-mail: ramos@ufpa.br https://orcid.org/0000-0002-2803-3248

${ }^{3}$ Faculdade de Matemática, Universidade Federal do Pará, R. Raimundo Santana Cruz, s/n, 68721-000, Salinópolis, PA, Brasil - E-mail: lindomar@ufpa.br https://orcid.org/0000-0002-0299-9298

${ }^{4}$ Faculdade de Matemática, Universidade Federal do Pará, R. Raimundo Santana Cruz, s/n, 68721-000, Salinópolis, PA, Brasil - E-mail: danilopaixao3@gmail.com https://orcid.org/0000-0003-1901-7083
} 
em que $\phi=\phi(x, t)$ é a variável de fase, $\delta G / \delta \phi$ é a derivada funcional da energia livre em relação à variável de fase, $M$ é a mobilidade da interface (ou a facilidade com que $\phi$ varia com o tempo) garante a evolução do sistema (1.1). Em certas ocasiões, a mobilidade é considerada anisotrópica, como adotado por [4], mas também pode ser considerada como uma constante (isotrópica), como adotado por [5].

Usando o modelo de Allen-Cahn, Xinfu Chen [2] considerou o seguinte problema de valor inicial

$$
\left\{\begin{array}{l}
u_{t}^{\varepsilon}=\varepsilon^{2} u_{x x}^{\varepsilon}-f\left(u^{\varepsilon}\right), \quad x \in \mathbb{R}, t>0 \\
u^{\varepsilon}(x, 0)=u_{0}(x), \quad x \in \mathbb{R}
\end{array}\right.
$$

relacionado com o estudo de movimentos no limite de fase de materiais cristalinos, genética de população e também com a propagação de pulsos nervosos, onde $\varepsilon>0$ é um pequeno parâmetro e $f(\cdot)$ é uma função suave tendo exatamente três zeros $\{-1,0,1\}$ e satisfazendo $f^{\prime}( \pm 1)>0, f^{\prime}(0)<0$ e $\int_{-1}^{1} f(s) d s=0$. Um exemplo típico de $f$ é a função $f(s)=s^{3}-s$. Na dinâmica do problema estudado pelos autores, foram observados quatro estágios. No primeiro estágio, observa-se a fase de separação com ordem de grandeza $O(|\ln \varepsilon|)$, no segundo estágio, nota-se a geração de padrões metaestáveis de ordem $O\left(\varepsilon^{-1}\right)$, no terceiro, a propagação em movimento super câmera lenta das interfaces, é de ordem $O\left(e^{l / \varepsilon}\right)$ e por último, a fase de aniquilação da interface é de ordem $O(1)$.

Em [6], Uzunca e Karasozen consideraram a equação de Allen-Cahn dada por

$$
u_{t}=\xi \Delta u-f(u), \quad(x, t) \in \Omega \times(0, T],
$$

onde $\xi>0$ é uma constante que define a espessura da interface e $\Omega \cup I R^{d}(d=1,2)$ é um domínio limitado. A norma em $L^{2}$ é induzida por

$$
u_{t}=-\frac{\delta \mathscr{E}(u)}{\delta u}
$$

caracterizada pela minimização do funcional energia de Ginzburg-Landau

$$
\mathscr{E}(u):=\int_{\Omega}\left(\frac{\xi}{2}|\Delta u|^{2}+F(u)\right) d x,
$$

com potencial $F(u)$. Segundo os autores a principal característica da equação de Allen-Cahn (1.3) é a rápida formação das camadas transientes e a formação exponencialmente lenta das camadas terminais para valores muito pequenos de $\xi$. Isto é interessante porque nos faz pensar na possibilidade de existir um limite inferior para $\xi$. Portanto, baseado nesta afirmação fazemos o seguinte questionamento:

"Existe uma constante c $>0$ tal que a taxa do decaimento exponencial da energia da equação de Allen-Cahn (1.3) enfraquece quando $\xi \rightarrow c$ ?”

Acerca deste questionamento, ressaltamos que não temos conhecimento de nenhum trabalho na literatura que faça uma investigação nesta direção e muito menos acerca do valor limite de $\xi$ 
que enfraqueça a taxa de decaimento exponencial da energia total associada à equação de AllenCahn. Outro fato que também merece ser investigado é como explicitar uma solução exata para tal problema.

É bem conhecido que em problemas lineares a solução exata pode ser obtida em muitos casos pelo método da separação de variáveis [3], no entanto, em problemas não lineares são raros os casos em que conseguimos explicitar a solução exata.

Para o caso de soluções exatas de problemas não lineares destacamos o trabalho de Zemskov e Loskuto [7]. Neste artigo os autores estudaram a equação de Fisher-Kolmogorov dada por

$$
u_{t}-D u_{x x}-\left(a-\frac{u}{\cos x}\right) u=0, \quad \text { com } a>0 .
$$

Usando o método da separação de variáveis e supondo $u(x, t)=X(x) T(t)$, os autores encontraram as equações diferenciais ordinárias dadas por

$$
T_{t}-q T+T^{2}=0 \quad \text { e } \quad D X_{x x}-(q-a) X=0,
$$

onde $q \in I R$ é o parâmetro de separação desconhecido. Eles obtiveram as soluções gerais da forma

$$
T(t)=\left\{\begin{array}{l}
\frac{q}{1+e^{c_{0}-q t}}, \quad \text { se } \quad q \neq 0, \\
\frac{1}{c_{0}-t}, \quad \text { se } \quad q=0
\end{array}\right.
$$

e ainda,

$$
X(x)=\left\{\begin{array}{l}
A_{1} e^{\lambda_{1} x}+A_{2} e^{\lambda_{2} x} \text { com } \lambda_{1,2}= \pm \sqrt{(q-a) / D} \text { se } q>a \\
B_{1} \cos (\lambda x)+B_{2} \operatorname{sen}(\lambda x) \text { com } \lambda=\sqrt{(a-q) / D} \text { se } q<a, \\
C_{1} x+C_{2}, \text { se } q=a,
\end{array}\right.
$$

onde a constante $c_{0}$ é definida pelas condições iniciais. Com isto, eles mostraram que a solução exata do problema é dada por

$$
u(x, t)=\left\{\begin{array}{lllll}
\frac{(a-D) \cos x}{1+e^{\left[c_{0}-(a-D)\right] t},} & \text { se } & q=a-D & \text { com } & q<a \\
\frac{(a+D) \cosh x}{1+e^{\left[c_{0}-(a+D)\right] t}}, & \text { se } & q=a+D & \text { com } & q>a
\end{array}\right.
$$

Na Figura 1 observamos as soluções exatas referentes às equações dadas em (1.10).

Analisando a Figura $1(a)$ percebemos que a solução exata se estabiliza no zero quando $t \rightarrow$ $-\infty$. Mas por outro lado, quando $t \rightarrow \infty$ notamos a presença de oscilações espaciais (perda de homogeneidade espacial). 


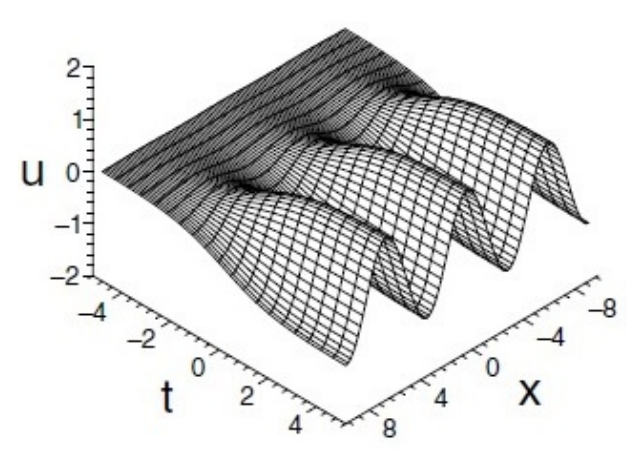

(a)

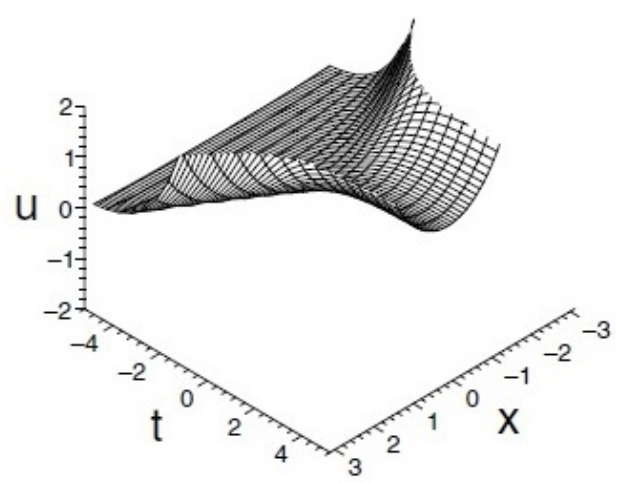

(b)

Figura 1: Solução exata com $c_{0}=0$ e $a-D=1$ (lado esquerdo) e com $c_{0}=0$ e $a+D=1$ (lado direito). Retirada de [7].

Para entendermos melhor o comportamento assintótico da solução encontrada por Zemskov e Loskuto em [7], fizemos uma análise baseada em argumentos de energia considerando o domínio compacto $[0, L]$. Definindo a energia total de (1.6) por

$$
E(t):=\frac{1}{2} \int_{0}^{L}|u|^{2} d x
$$

notamos que a mesma satisfaz a taxa de variação

$$
\frac{d}{d t} E(t)=-D \int_{0}^{L}\left|u_{x}\right|^{2} d x+a \int_{0}^{L}|u|^{2} d x-\int_{0}^{L} \frac{u^{3}}{\cos x} d x .
$$

Com isto, percebemos que a taxa de variação da energia não possui sinal definido, ou seja, temos uma "competição" entre as constantes $a>0$ e $D>0$ que altera qualitativamente o comportamento do modelo quando $t \rightarrow \pm \infty$.

Motivado pelos trabalhos mencionados anteriormente, consideramos a equação de Allen-Cahn unidimensional envolvida num problema que descreve a fase de uma mistura binária

$$
\left\{\begin{array}{l}
u_{t}-\xi^{2} u_{x x}-u\left(1-\frac{u^{2}}{k(x)}\right)=0 \quad \text { em } \quad(0,1) \times(0, \infty) \\
u_{x}(0, t)=u_{x}(1, t)=0, \quad \forall t \geq 0 \\
u(x, 0)=u_{0}(x), \quad 0 \leq x \leq 1
\end{array}\right.
$$

onde $k(x)>0$ é o coeficiente de reação. A variável de fase $u=u(x, t)$ denota a concentração da mistura e o parâmetro $\xi>0$ está relacionado à espessura da interface capturando o efeito dominante da cinética de reação.

O trabalho está organizado da seguinte forma: $\mathrm{Na}$ Seção 2, apresentamos o problema objeto de estudo, seguido do resultado que apresenta a solução exata. Na Seção 3, apresentamos a abordagem de energia do problema e os resultados de estabilização. Por fim, na Seção 4 apresentamos algumas simulações computacionais da solução exata. 


\section{EQUAÇÃO DE ALLEN-CAHN}

Nesta seção apresentamos o primeiro resultado deste trabalho que é a solução exata do problema (1.13).

\subsection{Solução exata do problema}

Consideramos o problema de difusão não linear dado por

$$
u_{t}-\xi^{2} u_{x x}-u\left(1-\frac{u^{2}}{k(x)}\right)=0 \quad \text { em } \quad(0,1) \times(0, \infty),
$$

onde $0<k(x) \leq 1$ é o coeficiente de reação determinado posteriormente. O termo de reação não linear dado por

$$
f(x, s):=s\left(1-\frac{s^{2}}{k(x)}\right),
$$

é o potencial de energia que conduz a solução ao estado $u=0$. As condições de contorno de Neumann homogêneas são dadas por

$$
u_{x}(0, t)=u_{x}(1, t)=0, \quad \forall t \geq 0,
$$

e a condição inicial por

$$
u(x, 0)=u_{0}(x), \quad 0 \leq x \leq 1 .
$$

Na proposição seguinte apresentamos a solução exata do problema em séries de Fourier.

Proposição 2.1. A solução exata do problema (2.1)-(2.4) $\operatorname{com} k(x)=\cos ^{2}(n \pi x)(n \in I N)$ é dada em série de Fourier por

$$
u(x, t)=\sum_{n=1}^{\infty} c_{n} \frac{e^{-\left(\xi^{2} n^{2} \pi^{2}-1\right) t}}{\sqrt{1+\frac{G_{0}^{2}}{\xi^{2} n^{2} \pi^{2}-1}\left(1-e^{-2\left(\xi^{2} n^{2} \pi^{2}-1\right) t}\right)}} \cos (n \pi x), \quad \xi>1 / \pi,
$$

onde $c_{n}$ é o coeficiente de Fourier dado por

$$
c_{n}=2 \int_{0}^{1} u_{0}(x) \cos (n \pi x) d x .
$$

Proof. Considerando o ansatz da forma

$$
u(x, t)=F(x) G(t)
$$

segue que

$$
\frac{\xi^{2} F^{\prime \prime}(x)+F(x)}{F(x)}=\frac{G^{\prime}(t)+\frac{1}{k(x)} F^{2}(x) G^{3}(t)}{G(t)} .
$$


Escolhendo $k(x):=F^{2}(x)$ ficamos com

$$
\frac{\xi^{2} F^{\prime \prime}(x)+F(x)}{F(x)}=\frac{G^{\prime}(t)+G^{3}(t)}{G(t)} .
$$

Observamos que o lado esquerdo da equação acima depende apenas de $x$, enquanto que o lado direito depende apenas de $t$. Logo podemos concluir que ambos os lados, são independentes de $x$ e $t$. Isto quer dizer que,

$$
\frac{\xi^{2} F^{\prime \prime}(x)+F(x)}{F(x)}=\frac{G^{\prime}(t)+G^{3}(t)}{G(t)}=\sigma, \text { para algum } \quad \sigma \in I R .
$$

Por outro lado, substituindo $u(x, t)=F(x) G(t)$ nas condições de contorno $u_{x}(0, t)=u_{x}(1, t)=0$ temos,

$$
\left\{\begin{array}{l}
u_{x}(0, t)=0 \quad \Rightarrow \quad F^{\prime}(0) G(t)=0 \quad \Rightarrow \quad F^{\prime}(0)=0, \\
u_{x}(1, t)=0 \quad \Rightarrow \quad F^{\prime}(1) G(t)=0 \quad \Rightarrow \quad F^{\prime}(1)=0,
\end{array}\right.
$$

e substituindo na condição inicial $u(x, 0)=u_{0}(x)$ temos

$$
u(x, 0)=u_{0}(x) \quad \Rightarrow \quad F(x) G(0)=u_{0}(x),
$$

de onde adotamos $G(0)=G_{0} \in I R_{*}$.

Com isto, passamos a considerar o problema de valor de contorno

$$
\left\{\begin{array}{l}
\xi^{2} F^{\prime \prime}(x)+(1-\sigma) F(x)=0 \quad \text { em } \quad(0,1), \\
F^{\prime}(0)=F^{\prime}(1)=0
\end{array}\right.
$$

e o problema de valor inicial

$$
\left\{\begin{array}{l}
G^{\prime}(t)-\sigma G(t)+G^{3}(t)=0, \quad \forall t \geq 0 \\
G(0)=G_{0}
\end{array}\right.
$$

Primeiramente revolvemos o problema de valor inicial. Para isto, linearizamos a equação em (2.14) multiplicando por $G(t)^{-3}$. Com isto, obtemos

$$
G(t)^{-3} G^{\prime}(t)=\sigma G(t)^{-2}-1 .
$$

Considerando a mudança de variável $y(t):=G(t)^{-2}$ temos

$$
y(t)^{\prime}=-2 G(t)^{-3} G^{\prime}(t) .
$$

Logo a equação (2.15) pode ser reescrita na forma linear

$$
y^{\prime}(t)=-2 \sigma y(t)+2 .
$$

Resolvendo a equação acima temos

$$
\begin{aligned}
y & =e^{-2 \sigma \int d t}\left(k+2 \int e^{2 \sigma \int d t} d t\right)=e^{-2 \sigma t}\left(k+2 \int e^{2 \sigma t} d t\right), \\
& =e^{-2 \sigma t}\left(k+\frac{e^{2 \sigma t}}{\sigma}\right)=k e^{-2 \sigma t}+\frac{1}{\sigma}=\frac{k \sigma e^{-2 \sigma t}+1}{\sigma} .
\end{aligned}
$$


Retornando à variável $G(t)$ obtemos

$$
G(t)^{-2}=\frac{k \sigma e^{-2 \sigma t}+1}{\sigma} \Rightarrow G(t)= \pm \sqrt{\frac{\sigma}{k \sigma e^{-2 \sigma t}+1}} .
$$

Para $t=0$ temos $G(0)=G_{0}$. Logo,

$$
G(0)= \pm \sqrt{\frac{\sigma}{k \sigma+1}} \Rightarrow k \sigma=\frac{\sigma-G_{0}^{2}}{G_{0}^{2}} .
$$

Substituindo (2.20) em (2.19) temos

$$
\begin{aligned}
G(t) & = \pm \sqrt{\frac{\sigma G_{0}^{2}}{\left(\sigma-G_{0}^{2}\right) e^{-2 \sigma t}+G_{0}^{2}}}= \pm \frac{G_{0}}{\sqrt{\left(1-\frac{G_{0}^{2}}{\sigma}\right) e^{-2 \sigma t}+\frac{G_{0}^{2}}{\sigma}}} \\
& = \pm \frac{G_{0}}{\sqrt{e^{-2 \sigma t}-e^{-2 \sigma t}\left(1-e^{2 \sigma t}\right) \frac{G_{0}^{2}}{\sigma}}}
\end{aligned}
$$

Finalmente obtemos que

$$
G(t)= \pm \frac{G_{0} e^{\sigma t}}{\sqrt{1-\frac{G_{0}^{2}}{\sigma}\left(1-e^{2 \sigma t}\right)}} \text { com } t \geq 0
$$

Por outro lado, para resolvermos o problema de contorno (2.13) precisamos analisar três casos.

Caso 1: Supondo $1-\sigma>0$ e considerando $F(x)=e^{\mu x}$ temos a equação característica

$$
\mu^{2} \xi^{2}+(1-\sigma)=0 \Rightarrow \mu= \pm i \frac{\sqrt{1-\sigma}}{\xi} \text {. }
$$

Segue daí que

$$
F(x)=e^{i \frac{\sqrt{1-\sigma}}{\xi} x} \text { e } F(x)=e^{-i \frac{\sqrt{1-\sigma}}{\xi} x}
$$

Pelo Princípio da Superposição temos que

$$
F(x)=c_{1} e^{i \frac{\sqrt{1-\sigma}}{\xi} x}+c_{2} e^{-i \frac{\sqrt{1-\sigma}}{\xi} x}, \quad c_{i} \in I R,
$$

também é solução do problema. Usando a relação de Euler $e^{ \pm i \theta}=\cos \theta \pm i \operatorname{sen} \theta$ podemos escrever

$$
F(x)=C_{1} \cos \left(\frac{\sqrt{1-\sigma}}{\xi} x\right)+C_{2} \operatorname{sen}\left(\frac{\sqrt{1-\sigma}}{\xi} x\right), \quad C_{i} \in \mathbb{C} .
$$

Segue das condições de contorno $F^{\prime}(0)=F^{\prime}(1)=0$ que

$$
\left\{\begin{array}{l}
F^{\prime}(0)=-C_{1} \frac{\sqrt{1-\sigma}}{\xi} \operatorname{sen}(0)+C_{2} \frac{\sqrt{1-\sigma}}{\xi} \cos (0)=0 \quad \Rightarrow \quad C_{2}=0 \\
F^{\prime}(1)=-C_{1} \frac{\sqrt{1-\sigma}}{\xi} \operatorname{sen}\left(\frac{\sqrt{1-\sigma}}{\xi}\right)=0 \Rightarrow C_{1}=0 \quad \text { ou } \quad \operatorname{sen}\left(\frac{\sqrt{1-\sigma}}{\xi}\right)=0 .
\end{array}\right.
$$


Como não estamos interessados na solução trivial, devemos considerar $C_{1} \neq 0$. Com isto obtemos a equação

$$
\operatorname{sen}\left(\frac{\sqrt{1-\sigma}}{\xi}\right)=0 \Rightarrow \sigma_{n}=1-\xi^{2} n^{2} \pi^{2}, \quad \forall n \in I N
$$

Logo temos a solução

$$
F_{n}(x)=C_{1} \cos (n \pi x), \quad \forall n \in I N
$$

Consequentemente, obtemos o coeficiente de reação

$$
k_{n}(x)=\cos ^{2}(n \pi x) \quad \text { com } \quad x \in(0,1), \quad \forall n \in I N,
$$

que é uma condição suficiente para usarmos o método da separação de variáveis.

Combinando as equações (2.23) e (2.29) e usando o Princípio da Superposição temos que

$$
u(x, t)=\sum_{n=1}^{\infty} c_{n} \frac{e^{-\left(\xi^{2} n^{2} \pi^{2}-1\right) t}}{\sqrt{1+\frac{G_{0}^{2}}{\xi^{2} n^{2} \pi^{2}-1}\left(1-e^{-2\left(\xi^{2} n^{2} \pi^{2}-1\right) t}\right)}} \cos (n \pi x), \quad \xi>1 / \pi
$$

onde $G_{0} \neq 0$ é constante e os coeficiente de Fourier $c_{n}(n=1,2, \ldots)$ são escolhidos de modo que tomando $t=0$ em (2.31) tenhamos

$$
u_{0}(x)=\sum_{n=1}^{\infty} c_{n} \cos (n \pi x), \quad \forall x \in[0,1] .
$$

Portanto os $c_{n}(n=1,2, \ldots)$ devem ser coeficientes de Fourier da função $u_{0}(x)$, dada em $[0,1]$. Note que $u_{0}(x)$ deve se escolhida de modo a ser uma função par e periódica de período 2, a fim de termos uma série de cossenos de argumento $n \pi$.

Para encontrarmos os valores de $c_{n}$, multiplicamos a equação (2.32) por $\cos (k \pi x)(k \in I N) \mathrm{e}$ integramos em $[0,1]$. Com isto, obtemos

$$
\int_{0}^{1} u_{0}(x) \cos (k \pi x) d x=\sum_{n=1}^{\infty} c_{n} \int_{0}^{1} \cos (n \pi x) \cos (k \pi x) d x
$$

Usando as relações de ortogonalidade entre as funções $\{\cos (n \pi x), \cos (k \pi x)\}$ temos

$$
c_{n}=2 \int_{0}^{1} u_{0}(x) \cos (n \pi x) d x, \quad \forall n \in I N .
$$

Caso 2: Supondo $1-\sigma=0$ o problema de contorno é dado por

$$
\left\{\begin{array}{l}
F^{\prime \prime}(x)=0 \text { em } \quad(0,1) \\
F^{\prime}(0)=F^{\prime}(1)=0
\end{array}\right.
$$


Segue daí que $F(x)=C_{1} x+C_{2}$ e devido as condições de contorno $F^{\prime}(0)=F^{\prime}(1)=0$ temos

$$
F^{\prime}(x)=C_{1} \quad \Rightarrow \quad F^{\prime}(0)=C_{1}=0 \quad \Rightarrow \quad C_{1}=0 .
$$

Logo temos que $F$ é uma solução constante dada por

$$
F(x)=C_{2}, \quad \forall x \in(0,1) .
$$

Como não estamos interessados na solução constante para $F$, o parâmetro $1-\sigma=0$ não serve.

Caso 3: Supondo $1-\sigma<0$ temos a equação característica

$$
\mu^{2} \xi^{2}+(1-\sigma)=0 \Rightarrow \mu= \pm \frac{\sqrt{\sigma-1}}{\xi} .
$$

Segue do Princípio da Superposição que

$$
F(x)=c_{1} e^{\frac{\sqrt{\sigma-1}}{\xi} x}+c_{2} e^{-\frac{\sqrt{\sigma-1}}{\xi} x}
$$

é uma solução do problema. Considerando a relação trigonométrica $e^{ \pm \theta}=\cosh \theta \pm \operatorname{senh} \theta$ temos que

$$
F(x)=C_{1} \cosh \left(\frac{\sqrt{\sigma-1}}{\xi} x\right)+C_{2} \operatorname{senh}\left(\frac{\sqrt{\sigma-1}}{\xi} x\right)
$$

Por outro lado, usando as condições de contorno $F^{\prime}(0)=F^{\prime}(1)=0$ temos que

$$
\left\{\begin{array}{l}
F^{\prime}(0)=C_{1} \frac{\sqrt{\sigma-1}}{\xi} \operatorname{senh}(0)+C_{2} \frac{\sqrt{\sigma-1}}{\xi} \cosh (0)=0 \quad \Rightarrow \quad C_{2}=0, \\
F^{\prime}(1)=C_{1} \frac{\sqrt{\sigma-1}}{\xi} \operatorname{senh}\left(\frac{\sqrt{\sigma-1}}{\xi}\right)=0 .
\end{array}\right.
$$

Como estamos interessados na solução não trivial, devemos considerar $C_{1} \neq 0$. Com isto temos a equação

$$
\operatorname{senh}\left(\frac{\sqrt{\sigma-1}}{\xi}\right)=0 \Rightarrow \sigma=1 .
$$

Com $\sigma=1$ temos a solução constante

$$
F(x)=C_{1}, \quad \forall x \in(0,1)
$$

Como não estamos interessados na solução constante para $F$, o parâmetro $1-\sigma<0$ não serve. Portanto a solução $u(x, t)$ do problema (2.1)-(2.4) é dada em (2.31). 


\section{ABORDAGEM DE ENERGIA}

Esta seção é dedicada às questões referentes à estabilização assintótica da equação de AllenCahn (2.1)-(2.4). Utilizamos o método multiplicativo para construir dois funcionais de energia. O primeiro funcional é denominado energia de Ginzburg-Landau e é responsável pela dinâmica de transição entre as fases, o segundo, denominado energia total, é responsável pela dinâmica global do sistema. Estes resultados são tratados nas duas proposições seguintes.

Proposição 3.2. A energia de Ginzburg-Landau do problema (2.1)-(2.4) é dada por

$$
\mathscr{G}(t):=\int_{0}^{1}\left(\frac{\xi^{2}}{2}\left|u_{x}\right|^{2}+f(u, x)\right) d x
$$

onde $f(u, x):=\frac{k(x)}{4}\left(1-u^{2} / k(x)\right)^{2}$, satisfaz a lei de dissipação

$$
\frac{d \mathscr{G}(t)}{d t}=-\int_{0}^{1}\left|u_{t}\right|^{2} d x
$$

Proof. Multiplicando a equação (2.1) por $u_{t}$ e integrando por partes em $[0, L]$ temos

$$
\int_{0}^{1}\left|u_{t}\right|^{2} d x+\xi^{2} \int_{0}^{1} u_{x} u_{x t} d x-\int_{0}^{1} u_{t} u d x+\int_{0}^{1} \frac{1}{k(x)} u_{t} u^{3} d x=0 .
$$

Consequentemente,

$$
\int_{0}^{1}\left|u_{t}\right|^{2} d x+\frac{\xi^{2}}{2} \frac{d}{d t} \int_{0}^{1}\left|u_{x}\right|^{2} d x-\frac{1}{2} \frac{d}{d t} \int_{0}^{1}|u|^{2} d x+\frac{1}{4} \frac{d}{d t} \int_{0}^{1} \frac{1}{k(x)}|u|^{4} d x=0 .
$$

Desde que consideremos a identidade

$$
-\frac{1}{2} \frac{d}{d t} \int_{0}^{1}|u|^{2} d x+\frac{1}{4} \frac{d}{d t} \int_{0}^{1} \frac{1}{k(x)}|u|^{4} d x=\frac{d}{d t}\left[\int_{0}^{1} \frac{k(x)}{4}\left(1-\frac{u^{2}}{k(x)}\right)^{2} d x\right],
$$

temos que

$$
\frac{d}{d t} \int_{0}^{1}\left(\frac{\xi^{2}}{2}\left|u_{x}\right|^{2}+f(u, x)\right) d x+\int_{0}^{1}\left|u_{t}\right|^{2} d x=0
$$

onde

$$
f(u, x):=\frac{k(x)}{4}\left(1-\frac{u^{2}}{k(x)}\right)^{2} .
$$

Assim, fica provado que a energia de Ginzburg-Landau associada ao problema (2.1)-(2.4) é dada por

$$
\mathscr{G}(t):=\int_{0}^{1}\left(\frac{\xi^{2}}{2}\left|u_{x}\right|^{2}+f(u, x)\right) d x
$$

e satisfaz a lei de dissipação

$$
\frac{d \mathscr{G}(t)}{d t}=-\int_{0}^{1}\left|u_{t}\right|^{2} d x
$$




\subsection{Estabilidade exponencial}

Aqui estudamos a estabilização das soluções do problema (2.1)-(2.4) através da energia total. Mais precisamente, provamos o decaimento exponencial quando $t \rightarrow \infty$.

Proposição 3.3. A energia total do problema (2.1)-(2.4) com $0<k(x) \leq 1$ é dada por

$$
E(t):=\frac{1}{2} \int_{0}^{1}|u|^{2} d x
$$

e satisfaz lei de dissipação

$$
\frac{d E(t)}{d t} \leq-\left(\frac{\xi^{2}}{c_{p}}-1\right) \int_{0}^{1}|u|^{2} d x-\int_{0}^{1}|u|^{4} d x,
$$

desde que $\xi \geq \sqrt{c_{p}}$, onde $c_{p}>0$ é a constante de Poincaré.

Proof. Multiplicando a equação (2.1) por $u$ e integrando por partes em $[0,1]$ temos

$$
\frac{1}{2} \frac{d}{d t} \int_{0}^{1}|u|^{2} d x+\xi^{2} \int_{0}^{1}\left|u_{x}\right|^{2} d x-\int_{0}^{1}|u|^{2} d x+\int_{0}^{1} \frac{1}{k(x)}|u|^{4} d x=0 .
$$

Definindo a energia total por

$$
E(t):=\frac{1}{2} \int_{0}^{1}|u|^{2} d x
$$

segue que

$$
\frac{d E(t)}{d t}=-\xi^{2} \int_{0}^{1}\left|u_{x}\right|^{2} d x+\int_{0}^{1}|u|^{2} d x-\int_{0}^{1} \frac{1}{k(x)}|u|^{4} d x
$$

Usando a desigualdade de Poincaré e levando em conta que $0<k(x) \leq 1$, temos

$$
\frac{d E(t)}{d t} \leq-\left(\frac{\xi^{2}}{c_{p}}-1\right) \int_{0}^{1}|u|^{2} d x-\int_{0}^{1}|u|^{4} d x,
$$

onde $c_{p}>0$ é a constante de Poincaré. Desde que $\xi \geq \sqrt{c_{p}}$, garantimos a lei de dissipação.

Teorema 3.1. A energia total do problema (2.1)-(2.4) com $0<k(x) \leq 1$ e $\xi>\sqrt{c_{p}}$, decai exponencialmente para zero com $t \rightarrow \infty$, isto é,

$$
E(t) \leq \frac{\left(\xi^{2}-c_{p}\right) E(0)}{\xi^{2}-c_{p}\left[1-2 E(0)\left(1-e^{-\frac{2}{c_{p}}\left(\xi^{2}-c_{p}\right) t}\right)\right]} e^{-\frac{2}{c_{p}}\left(\xi^{2}-c_{p}\right) t}, \quad \forall t>0,
$$

onde $c_{p}>0$ é a constante de Poincaré.

Proof. Inicialmente consideramos a lei de dissipação dada na Proposição (3.3)

$$
\frac{d E(t)}{d t} \leq-\left(\frac{\xi^{2}}{c_{p}}-1\right) \int_{0}^{1}|u|^{2} d x-\int_{0}^{1}|u|^{4} d x .
$$


Por outro lado, considerando a desigualdade de Jensen

$$
g\left(\int_{0}^{1} v(x) d x\right) \leq \int_{0}^{1} g(v(x)) d x
$$

com $g(x)=x^{2}$ e $v(x)=|u|^{2}$, é fácil ver que $g^{\prime \prime}>0 \mathrm{e}$

$$
\left(\int_{0}^{1}|u|^{2} d x\right)^{2} \leq \int_{0}^{1}|u|^{4} d x
$$

Consequentemente podemos escrever

$$
\frac{d E(t)}{d t} \leq-2\left(\frac{\xi^{2}}{c_{p}}-1\right) E(t)-4 E^{2}(t) .
$$

Para encontrarmos $E(t)$ na desigualdade acima, multiplicamos ambos os lados por $[E(t)]^{-2}$. Com isto, obtemos

$$
\frac{d E(t)}{d t}[E(t)]^{-2} \leq-2\left(\frac{\xi^{2}}{c_{p}}-1\right)[E(t)]^{-1}-4
$$

Fazendo a mudança de variável

$$
u(t):=-[E(t)]^{-1} \Rightarrow \frac{d}{d t} u(t)=\frac{d E(t)}{d t}[E(t)]^{-2},
$$

e substituindo em (3.21) temos

$$
\frac{d u(t)}{d t} \leq 2\left(\frac{\xi^{2}}{c_{p}}-1\right) u(t)-4, \forall t>0 .
$$

Usando a desigualdade de Gronwall obtemos

$$
u(t) \leq\left(u(0)-\frac{2 c_{p}}{\xi^{2}-c_{p}}\right) e^{\frac{2}{c_{p}}\left(\xi^{2}-c_{p}\right) t}+\frac{2 c_{p}}{\xi^{2}-c_{p}} .
$$

Em seguinda, de $u(t)=-[E(t)]^{-1}$ obtemos $u(0)=-[E(0)]^{-1}$. Consequentemente,

$$
\begin{aligned}
E(t) & \leq \frac{\xi^{2}-c_{p}}{\left[2 c_{p}-\left(\xi^{2}-c_{p}\right) u(0)\right] e^{\frac{2}{c_{p}}\left(\xi^{2}-c_{p}\right) t}-2 c_{p}} \\
& =\frac{\left(\xi^{2}-c_{p}\right) E(0)}{\left[2 c_{p} E(0)+\left(\xi^{2}-c_{p}\right)\right] e^{\frac{2}{c_{p}}\left(\xi^{2}-c_{p}\right) t}-2 c_{p} E(0)} \\
& =\frac{\left(\xi^{2}-c_{p}\right) E(0)}{\xi^{2}-c_{p}+2 c_{p} E(0)\left(1-e^{-\frac{2}{c_{p}}\left(\xi^{2}-c_{p}\right) t}\right)} e^{-\frac{2}{c_{p}}\left(\xi^{2}-c_{p}\right) t}
\end{aligned}
$$

Portanto obtemos

$$
E(t) \leq \frac{\left(\xi^{2}-c_{p}\right) E(0)}{\xi^{2}-c_{p}\left[1-2 E(0)\left(1-e^{-\frac{2}{c_{p}}\left(\xi^{2}-c_{p}\right) t}\right)\right]} e^{-\frac{2}{c_{p}}\left(\xi^{2}-c_{p}\right) t}
$$

onde $\xi^{2}>c_{p}$ e $c_{p}>0$ é a constante de Poincaré. Assim fica provado que a energia total do problema (2.1)-(2.4) decai exponencialmente para zero com $t \rightarrow \infty$. 
Agora, chamamos atenção para o questionamento feito na introdução.

"Existe uma constante $c>0$ tal que a taxa do decaimento exponencial da energia da equação de Allen-Cahn (1.3) enfraquece quando $\xi \rightarrow c$ ?”

Ressaltamos que a resposta é sim, e além disso, podemos afirmar pela Proposição 2.1 e pelo Teorema 3.1 que

$$
c>c_{0}:=\max \left\{\frac{1}{\pi}, \sqrt{c_{p}}\right\} \approx \max \left\{0,3183, \sqrt{c_{p}}\right\} .
$$

Na seção seguinte, veremos como se comporta a energia total do problema quando $\xi \rightarrow c$ não obedece a limite (3.27).

\section{SIMULAÇÃO COMPUTACIONAL}

Para realizarmos as simulações computacionais da solução exata e da energia total do problema (2.1)-(2.4), usamos o software MATLAB. Nossa intenção aqui é mostrar que o parâmetro $\xi$ responsável por definir a espessura da interface, afeta diretamente a taxa de decaimento exponencial da energia total e consequentemente, comprovar a afirmação dada em (3.27) que estabelece um limite inferior para $\xi$.

\subsection{Solução exata e energia total - Caso: $n=2$}

Neste caso consideramos os dados $T=0,1, G_{0}=1, n=2$ e assumimos os valores de $\xi=0,5$ satisfazendo (3.27) e $\xi=0,15$ que não satisfaz (3.27).
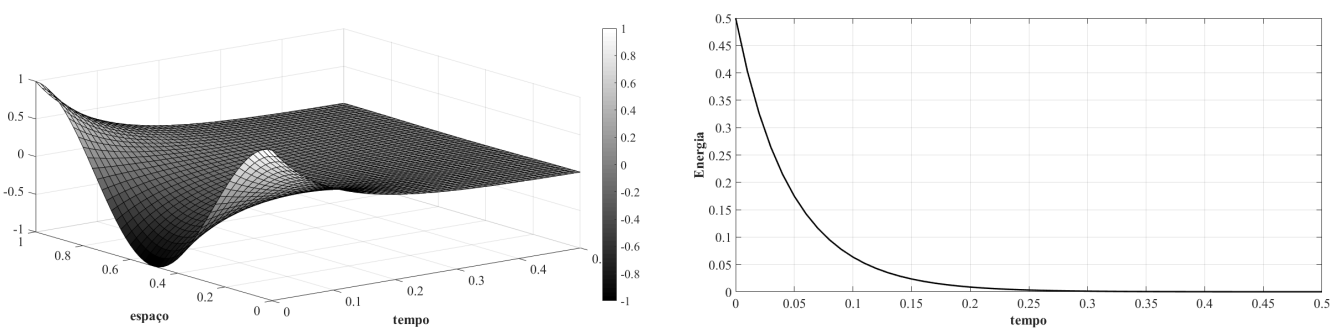

Figura 2: Solução exata e energia total, $\operatorname{com} \xi=0,5$ 

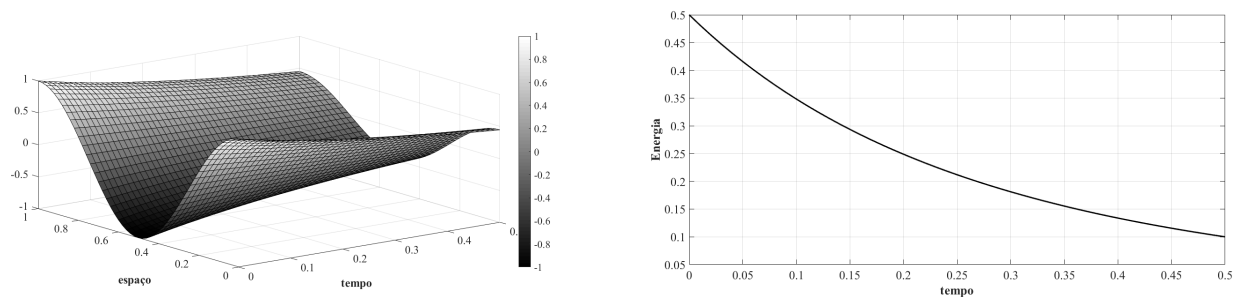

Figura 3: Solução exata e energia total, $\operatorname{com} \xi=0,15$

\subsection{Solução exata e energia total - Caso: $n=4$}

Aqui novamente consideramos os dados $T=0,1, G_{0}=1 \mathrm{com} n=4$ e assumimos os valores de $\xi=0,5$ e $\xi=0,15$ de modo que possamos comparar com (3.27).
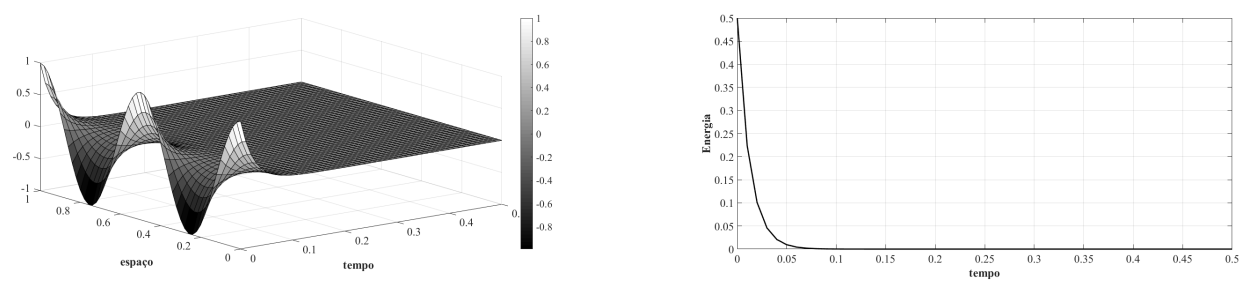

Figura 4: Solução exata e energia total, com $\xi=0,5$
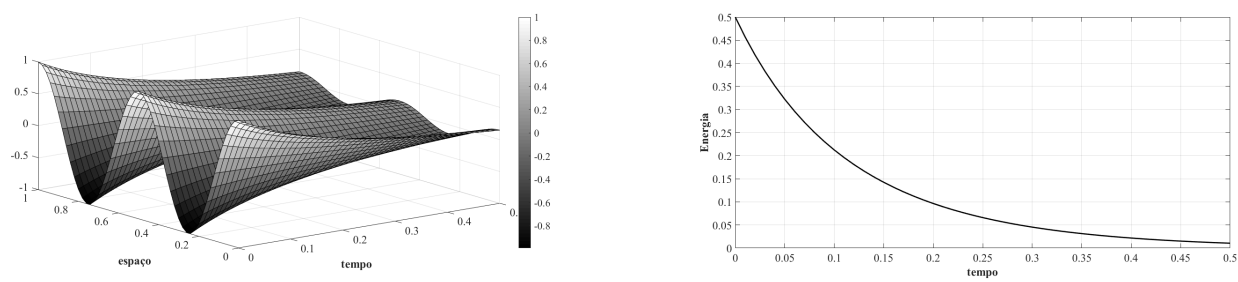

Figura 5: Solução exata e energia total, $\operatorname{com} \xi=0,15$

Comentário: Nas simulações realizadas acima percebemos que a solução exata e a energia total possuem comportamentos bem distintos dependendo do valor do coeficiente $\xi$. Nas Figuras 2 e 4 , no caso em que $\xi=0,5$, percebemos que a energia decai muito rápido para zero, enquanto que nas Figuras 3 e 5 com $\xi=0,15$ notamos o enfraquecimento da taxa de decaimento exponencial. A explicação matemática para isto se deve ao fato de

$$
\xi=0,15<\max \left\{0,3183, \sqrt{c_{p}}\right\},
$$

alterando assim, a taxa da exponencial de $e^{-\left(\xi^{2}-c_{p}\right) t}$. Por outro lado, do ponto de vista físico, passamos a entender melhor a dinâmica que leva a formação exponencialmente lenta das camadas terminais da interface para valores muito pequenos de $\xi$ (ver [6]). 


\title{
5 CONSIDERAÇÕES FINAIS
}

Neste trabalho foi apresentado uma solução exata para o modelo de Allen-Cahn por um método de separação de variáveis e um resultado de estabilização assintótica da energia total do modelo. Com isto, foi possível entender melhor o processo de formação exponencialmente lenta da camadas terminais da interface para valores muito pequenos de $\xi$. Até onde temos conhecimento, esta é a primeira vez que se estabelece uma conexão entre a estabilização exponencial do modelo de Allen-Cahn e o parâmetro de espessura das camadas.

\begin{abstract}
In this work we study some qualitative properties of the Allen-Cahn equation. This equation has been studied widely in several areas of science and mainly in the evolution of micro-structures during the solidification process of a pure metal or metallic alloy. The main results achieved are: the exact solution, the energy of Ginzburg-Landau and the exponential decay property of the total energy of the model. The exact solution of the problem was built from variables separation method thanks to a particular choice of the coefficient of reaction. In respect to the exponential stabilization of the total energy of solutions, we use the multiplicative techniques in order to establish the energy dissipation law and in the following we use classical inequalities of the mathematical analysis to build the estimate of exponential decay.
\end{abstract}

Keywords: diffusion equation, Allen-Cahn equation, exact solution, exponential stabilization.

\section{REFERÊNCIAS}

[1] S. Allen \& J. Cahn. A microscopic theory for antiphase boundary motion and its apllication to antiphase domain coaserning. Acta Metall, 27 (1979), 1084-1095.

[2] X. Chen. Generation, propagation, and annihilation of metastable patterns. Journal of Differential Equations, 206 (2004), 399-437.

[3] L.C. Evans. "Partial differential equations", volume 83. Wiley (1999), 185 p. doi:10.2307/3618751.

[4] A. Karma \& W. Rappel. Quantitative phase-field modeling of dendritic growth in two and three dimensions. Physical Review E Physics, 57 (1998), 4323-4349.

[5] R. Kobayashi. Modeling and numerical simulations of dendritic crystal growth. Physica D, 63(3-4) (1993), 410-423.

[6] M. Uzunca \& B. Karasozen. Energy Stable Model Order Reduction for the Allen-Cahn Equation. In "Model Reduction of Parametrized Systems". Springer (2017), p. 403-419. doi:10.1007/978-3-31958786-8_25.

[7] E.P. Zemskov \& A. Loskutov. Exact analytical solutions for nonlinear waves in the inhomogeneous Fisher-Kolmogorov equation. The European Physical Journal B, 79(1) (2010), 79-84. doi:10.1140/ epjb/e2010-90983-8. 